\title{
Corruption and Decentralization
}

\author{
Emanuela Carbonara* \\ Wadham College, University of Oxford \\ Dipartimento di Scienze Economiche, Università di Bologna ${ }^{\dagger}$ \\ September 1999
}

\begin{abstract}
This paper studies the effect of decentralization on corruption in a hierarchical organization, where decentralization is intended as the delegation of control power to lower levels in a hierarchy. Decentralization causes a loss in control to the higher levels, thus curbing their incentives to monitor and detect corrupt activities. However, it also lowers the expected gains from corruption as, following decentralization, the number of individuals who are in charge of a single decision is reduced. It is then more likely that corrupt agents are called to bear the consequences of their actions. Hence, decentralization, although creating agency problems inside an organization, can help in controlling corruption.
\end{abstract}

JEL Classification Numbers: D23, D73, D82.

Keywords: Corruption, Delegation, Formal Authority, Real Authority.

\footnotetext{
*This paper is a revised version of our earlier working paper "Bureaucracy, Corruption and Decentralization". I thank Philippe Aghion, Alan Beggs, Sudipto Bhattacharya, Gabriella Chiesa, Luigi Franzoni, Carlo Scarpa, Stefano Zamagni and seminar partecipants at the universities of Bologna and Oxford for useful comments.

${ }^{\dagger}$ Address for correspondence: Dipartimento di Scienze Economiche, 2 piazza Scaravilli, 40126 Bologna, Italy. Tel. +39 051209 8122. Fax. +39 0512098040 . e-mail: carbonara@economia.unibo.it.
} 


\section{Introduction}

Traditionally, the discretionary power held by members of an organization is regarded as one of the major sources of corrupt behavior. Klitgaard (1988) states that corruption depends positively on the amount of monopoly power and discretion enjoyed by the participants to an organization.

This way of addressing the issue does not consider that often individuals with more monopoly power are also the ones who bear the highest opportunity cost from corruption. Moreover, in the event that monopoly power allowed higher rents from corruption, a more powerful individual attracts also more attention from corruption detectors. All these elements can lead to situations where increasing discretion discourages corruption. Individuals holding power face a trade off, where they have to balance the increased opportunities (and therefore value) to be corrupt with the larger burden they hold.

Decentralisation transfers control power and discretion to lower hierarchical layers, thus leading them to consider such trade-off. As a result, the impact of decentralisation on the extent of corruption affecting an organisation depends on the direction towards which the trade off is resolved. The problem analyzed in this paper is how an organization should be designed to resolve the trade off in the sense of a reduction in corruption. ${ }^{1}$

As Aghion and Tirole (1997) point out effectively, there are two distinct sources of decisional power. Firstly, there is the power legally and explicitly attributed by means of a contract (control power or formal authority). ${ }^{2}$ Secondly, there is the power that derives from the knowledge of crucial information (real authority). By revealing such information strategically, members to an organisation can redirect the use of productive assets towards their own goals, even when they lack formal authority. However, centralized decision making certainly reduces the likelihood that subordinates are called to make a decision, which lowers the subordinates' incentives to work. ${ }^{3}$

The same is true for any superior layers that had the task to monitor subordinates' behaviour and detect corrupt activities. When the degree of decen-

\footnotetext{
${ }^{1}$ It is therefore assumed that corruption is harmful and should be minimised.This is in line with the most recent literature, which shows that corruption has highly indesirable effects on economic systems. It affects negatively the rate of growth of a country's GDP, the productivity of public investments and also the allocation of talent (see Mauro, 1995, Tanzi, 1998, Tanzi and Davoodi, 1997).

${ }^{2}$ Formal authority is the power to exert the residual rights of control, i.e. to decide in all the situations not contemplated by the initial, inevitably incomplete contract that links each member to the organization. This kind of power can be transferred from the owners to the subordinates by means of an act of delegation.

${ }^{3}$ This effect is what is called "basic trade off" in Aghion and Tirole (1997).
} 
tralization is high, superiors have little incentive to perform their screening and detection activities because they have little power to stop subordinates' decisions. Decentralization reduces the amount of monitoring, leaving local offices more freedom to pursue their corrupt activities. ${ }^{4}$

Decentralization has another important implication: it concentrates decisional powers in the hands of one individual, eliminating overlaps in competence and makes it easier to identify who took a certain decision. An agent operating at a low level of a hierarchy can always hope that the consequences of his corrupt act will not fall on his head if he is not the sole accountable for it. In fact, if superiors have formal authority upon that decision, the implementation of the agent's choice can be stopped. In other words, even if he has performed the illegal act he was bribed for (suggesting a bad project), the project can be rejected at higher levels and the agent will not bear the losses from his inefficient choice. This is not true anymore if he is in charge of the decision. Therefore, decentralization allows a more precise allocation of the consequences of corrupt acts. Agency problems, instead of being the main cause of corruption and agents' opportunistic behavior, under certain conditions can be helpful in alleviating them.

On the other hand, assume the reward for detectors is directly proportional to the amount of bribes they discover. ${ }^{5}$ If the manager benefits more from uncovering more bribes, decentralization can improve her incentives to monitor corrupt agents, thus increasing the probability that they are caught and the expected costs from corruption.

The paper is organized as follows. The next section describes the model. Section 3 characterizes the consequences of decentralization when only the lowest hierarchical layer is corruptible. Section 4 depicts the optimal anti corruption policy. Section 5 extends the model to include monetary incentives and punishment schemes and shows that the conclusions obtained are robust to the introduction of these "more traditional" incentive schemes. Section 6 considers the problem of a corrupt top bureaucrat. Section 7 adds a new dimension to the moral hazard problem, analyzing the case where the size of the bribe paid to agents increases with decentralisation. Section 8 concludes.

\footnotetext{
${ }^{4}$ In this sense, what stated by Rose-Ackerman (1978), that decentralization increases the risk of corruption since it limits review and detection, is confirmed. Decentralization decreases monitoring by superiors also because of another, more frequently cited, reason. It renders access to crucial information recorded by subordinates more difficult (it is more difficult to check their books and balances). However, while this problem can be overcome (albeit partially) by the introduction of accounting standards and transparency rules, weak incentives are more difficult to solve.

${ }^{5}$ This can either imply that detectors are allowed to keep a percentage of the bribes they retrieve or that, implicitly, their prestige and career inside the organisation are linked to the amount of corruption they discover.
} 


\subsection{Related Literature}

The positive relation between a member's monopoly power and the extent of corruption is stated also by Shleifer and Vishny (1993). When many officers are in charge of a decision, they exert a multiple veto power on each other, preventing the others from providing the corrupt service, reducing the "value" of their act. In their model, decentralization unambiguously increases corruption, eliminating this veto problem. Banerjee (1997) claims that public bureaucracies are affected by corruption, red tape and weak incentives just because governments acts to improve social welfare. Paradoxically, inefficiencies and corruption would not occur if the government were not interested in social welfare, since the bureaucrats, allocating scarce resources to those people who have the highest ability to pay, would not be acting illegally. ${ }^{6}$ Bac (1996) investigates the relation among the structure of hierarchies, the monitoring technology and the extent of internal and external corruption. Structure of hierarchies means the number of layers and the span of control for each layer and not the allocation of power, as in our model. He concludes that a flatter hierarchy is to be preferred when monitoring is not specialized (a given monitoring effort implies the same detection probability on all subordinates, which is equivalent to saying that there are economies of scale in monitoring), whereas a steeper structure reduces the risk of internal corruption, because the total amount of bribes that could be used to corrupt the manager is lower.

Other contributions have considered the interaction of corruption at different levels of a hierarchy. Cadot (1987) shows, in a dynamic model, how the combination of strong power - e.g. regulatory or granting permits - and low wages for public officers create "basic incentives" for corruption, conclusion in line with the observation of high levels of corruption in many Third World countries. Basu, Bhattacharya and Mishra (1992) consider that not only lower officers have the problem of being caught after accepting a bribe, but also their corrupt superiors. Corruption becomes a recursive problem. A good deterrence policy is that of increasing the probability that a corrupt officer is caught rather than stiffening the penalty imposed.

\footnotetext{
${ }^{6}$ As Banerjee (1997), also Acemoglu and Verdier (1997) point out that the crucial issues in explaining corruption are agency problems between the government and its bureaucrats and the inability of the government to make interventions without recurring to a bureaucracy. The opportunity for the bureaucrats to gain rents from corruption can lead to misallocation of talents (as these rents will attract not only those who are more able to perform a particular activity, but also others, who would perform better if assigned to other tasks).
} 


\section{The Model}

Consider an organization consisting of three hierarchical levels. The first layer is a principal, who lacks either the expertise or the time to perform the activity the organization has been set up for. She then delegates all her control power to the lower layers. The second hierarchical level is a central office (top manager or manager) who has the task to coordinate the activities performed by the agents and to monitor their behavior to detect corruption. The third level consists of $N$ local offices (agents), whose task is to screen a certain number of potential projects and to report information about them to the central office. Both the top bureaucrat and the local offices can be corrupt.

To minimize corruption, the principal decides on the allocation of control, choosing whether to delegate the power to select a project to the central office or to the local ones. In the first case the central office checks the information reported by the agents and decides which projects to implement. In the second case, which correspond to a more decentralized setting, this decision remains in the hands of the local offices and the superior layer performs only tasks of general supervision and detection of corruption.

Organizational Structure A hierarchy is formed by three layers.

1. A principal (either a private body, like the shareholders of a bank or of a firm, or a public institution, for instance a legislative body) and whose aim is to reduce corruption ${ }^{7}$. The principal decides the allocation of authority to the hierarchical layers below, chooses the penalties to impose on corrupt agents and the compensation policies for the managers who report cases of corruption.

2. An organization, consisting of a top manager ("she", also called "central office"), who controls and monitors a lower layer of $N$ agents.

3. The lower layer of $N$ agents, each (he) in charge of one productive activity and each representing a "local" or "sectorial" office. Agents are in direct contact with "clients" or "project proponent".

\footnotetext{
${ }^{7}$ In the framework considered here, there is no trade off between the subordinates' performance and the level of corruption. As a result, the principal's objective function is separable in performance and corruption and these two problems can be tackled independently of each other. This would not be true if, for istance, the subordinate's effort depended on the extent of corruption in the organization.
} 
The productive activity consists of selecting and realizing many different projects.

There are many institutions (either private or public) whose activity can be represented by the model depicted above. The most direct example is that of a financial intermediary or a bank. There are $N$ local offices or divisions, each specialized in a particular geographical area or in a given sector, who have to select projects to finance among many applicants. They screen the projects proposed and report the information they find to the central office. As there is always a risky component in a project, a negative outcome can be either the result of "bad luck" or of a "bad" project, therefore a local office can decide to accept a bribe from a project of low quality and report false information to the superior layer. Another example is that of a public institution who has to implement projects in a given area, for instance, street lighting or motorways. In order to implement these projects, the institution stipulates procurement contracts with external firms, who have to be selected, in principle by following criteria of economic efficiency. Again, local offices (plausibly those situated in the area which is going to benefit from the project) have to screen applicants, who can bribe them to be chosen.

The principal lacks either the expertise or the time to perform the productive activity directly and therefore has to delegate it. Before production starts, the principal chooses the structure of the organization, deciding the allocation of formal authority.

In that follows it will be assumed that the the agent has formal authority (decentralization) with probability $z$, whereas with probability $1-z$ the top manager is in control (centralization). Probably, the two most common cases are $z=0$ (full centralization) and $z=1$ (full decentralization), however, modeling the degree of decentralization as a continuous variable helps when comparative statics is performed. This procedure has no influence on the quality of the results, since a result verified for any $z$ must be true also for $z \in\{0,1\}$.

To interpret $z$, it could be imagined that the allocation of control depends on an external event, which occurs with probability $z$ and whose occurrence takes place or is observed only after the parties have exerted effort but before project implementation. ${ }^{8}$ It could also be imagined that each productive activity consists of a continuum of elementary decisions, to be taken simultaneously, so that $z$ represents the share of those decisions delegated to the agent. By taking these decisions according to his own interests, the agent can jeopardize the top manager's objectives and prevent her from taking certain

\footnotetext{
${ }^{8}$ This would be a case of contingent control in the spirit of Aghion and Bolton, (1992).
} 
actions and $z$ represents the share of veto powers in the agent's hands.

If the top manager has formal authority, she is in the position to overrule the agents and pursue her own goals. Conversely, if the agents have authority, they are in charge of the decisional process in the activity they control and the top manager cannot intervene to change their decisions, even if they are at odds with her personal interests. ${ }^{9}$

The principal can never be corrupt. This assumption is certainly plausible in case the organisation described were a private financial institution. It is hard to think that shareholders, for example, especially those in control of the majority stake, risked bankruptcy for a small fee. However, even in the case of public institutions, if the aim is the elimination if corruption, there must be some corruption free body, like, for example, the civil society. ${ }^{10}$

Initially, it will be assumed that only agents can be corrupt. This hypothesis will be removed later.

Projects At the beginning of the production process, each agent is randomly matched with a "client", who proposes a project.

There are either good or bad projects. Each type occurs with ex ante probability $\frac{1}{2}$.

Payoff from good projects. All good projects are equal. The top manager's profit from a good project is $G_{1}>0$, whereas the agent's payoff is $g_{1}>0$ and the principal's is $G$.

Payoff from bad projects. All bad projects yield $-G_{2}$ and $-g_{2}\left(G_{2}, g_{2}>\right.$ $0)$ respectively to the principal and to the agent. Their payoff thus is negative. In addition to that, bad projects also yield a loss $L$ to the principal. Without bribery, a bad project would never be chosen.

It is assumed here that the outcome of a project, by itself, does not constitute evidence of corruption. This can be justified by assuming that the

\footnotetext{
${ }^{9}$ This is the situation typically considered in the literature on agency. In that literature, delegation occurs because the principal has not either the time or the ability to undertake that activity. This explains why the agent can bias her decisions and actions toward his own goals, thus behaving with moral hazard. Either the principal lacks the information to check that the agent's decisions harm her interest (hidden information) or she is not able to observe the agent actions (hidden action). The result of this informational asymmetry is that the agent not only has the required formal authority to make decisions, as a consequence of delegation, but also the real authority.

${ }^{10}$ It has been assumed here that the highest layer in the hierarchy cannot be bribed. In case such hypothesis were released the organization would be shaped to maximise the principal gains. This case will be analysed in Chapter 3 .
} 
top manager needs evidence to sue an agent, even if she could infer from the project's outcome that corruption took place. ${ }^{11}$

Production If agents recognise a good project, they always suggest it. If the project is bad, instead, two cases can occur: if the agent is honest, he reports honestly what he found to the top manager, who then discards the project. If the agent is corrupt, he lies to the superior layer, stating that the project is good and suggesting its implementation.

The top manager verifies the reported information. This represents $e x$ ante monitoring in the sense of Aghion and Tirole (1997): the superior monitors to acquire information that allows her to evaluate the decisions taken by the subordinate. In case she did not agree and has formal authority she can impose a different course of action. It is therefore monitoring that takes place before the agent exerts his actions and not after, that would be a mere assessment of the results obtained. ${ }^{12}$

If the manager herself is corrupt, an agent may try to collude with her, either to avoid being monitored in the first place or not to be denouced if found out having suggested a bad project.

The flow of information goes upstream and screening is sequential. The superior layers have no opportunity to collect information directly because they have no direct contact with the projects and they can only verify the information reported by the subordinates.

The manager checks the information about one specific project with effort $e_{M}$ (where the subscript $M$ stays for manager) and learns with probability $e_{B}$ the true type of the project suggested, $e_{M} \in\left[\underline{e}_{M}, \bar{e}_{M}\right], 0<\underline{e}_{M}<\bar{e}_{M}<1$. She repeats the same activity for all recommended projects, bearing a cost equal to $c_{M}\left(N^{R} e_{M}\right)=N^{R} \frac{e_{M}^{2}}{2}$, where $N^{R}$ is the number of recommended projects.

The probability $1-e_{M}$ then measures the extent of the agent's real authority, because it is when such circumstances occur that, no matter who actually holds formal authority, the agent has full decisional power, being the other party uninformed.

If the agents have formal authority over the project choice, they choose

\footnotetext{
${ }^{11}$ Alternatively, it can be thought that the agent makes a mistake when assessing a project's quality. This feature can be introduced explicitly into the model by assuming that the agent observes a signal about the project's nature, signal correct with a given probability $\gamma>0$. It would always be possible that the project is bad even if the agent is honest and observing a bad outcome would not imply that bribery occurred. However, this complicates notation without changing the nature of results.

${ }^{12}$ This is the typical definition of monitoring in the incentive literature, ex post monitoring, where a more appropriate term would be auditing (see Baron and Besanko, 1984).
} 
whether to implement the project proposed by the client they have been matched with according to the information they have.

\section{Timing}

1. The principal chooses the allocation of control power in the organisation.

2. The agent is matched with a client and screens the project he offers.

3. If bad, the client offers the agent a bribe.

4. The agent decides whether to accept and then communicates to the top manager the information he has and, when he has formal authority, the projects he is going to implement.

5. The top manager screens the information obtained by all agents.

6. If the top manager discovers a bad, she reports the agent to the principal and receives a share $s$ of the bribe as a compensation. If the manager is informed and has formal authority (and is not corrupt), she stops the bad project. If the agent has formal authority, even when he has been caught, the bad project is implemented.

7. All good projects are implemented, plus unrecognized bad. All projects recommended are implemented if agents have formal authority.

The manager examines only the information the agent reports. This is therefore a case of sequential screening, where superiors screen only the projects not previously rejected by some lower layer. ${ }^{13}$ The agents have therefore a sort of filtering power, which make them an indispensable figure to have on one's side in the client's eyes.

In addition, only clients with bad projects bribe. This hypothesis is quite plausible as implementing a good is always desirable and, if the clients know the nature of the project they propose, they will probably try hard to demonstrate their nature. For example, if they are denied implementation after refusing to pay a bribe, they can denounce the agent. However, also a scenario where both project types bribe is possible. Agents could avoid to pronounce a clear rejection for a good project that refuses to pay and simply

\footnotetext{
${ }^{13}$ This model is therefore different in this respect from Aghion and Tirole (1997), who assume simultaneous screening. Moreover, the assumption of sequential screening implies that the top bureaucrat never has real authority, since production takes place only when the agent has information.
} 
delay implementation, until the client resolves to pay "to oil the wheels". To simplify the model, only bad offers a bribe, but the alternative hypothesis would not change substantially our results. ${ }^{14}$ The timing is depicted in Figure 1 .

Preferences and Utility Functions The manager and the agents are risk neutral. Agents can be either corrupt or honest. In general, here it is assumed that being corrupt is not an intrinsic quality of the agent but depends on the specific circumstances agents find themselves in. Each agent has a private-information cost $h$ of being corrupted, cost that measures the agent's degree of honesty: the higher $h$, the more honest the agent. If an agent is offered a bribe high enough, he becomes corrupt.

In order to describe the parties' utility functions it should be distinguished between the cases of non-corrupt and corrupt agents.

No corruption. If all agents are honest, only good projects are implemented. In this case there is perfect coincidence of interests between each agent and the top manager. ${ }^{15}$

Each agent has an expected payoff $U_{A}=\frac{g_{1}}{2}$, whereas the manager's total payoff is

$$
U_{M}=N\left[\frac{G_{1}}{2}-\frac{e_{M}^{2}}{2}\right]
$$

hence, the manager exerts only the minimum effort $\underline{e}_{M}$.

Corruption. In this case, both good and bad projects are suggested, bad provided that the clients who propose them offer the agents a bribe $b$ sufficiently high. The bribe is paid to the agent to report the wrong information. Corruption then means that a bad project is presented as a good to the superior. Moreover, the content of the information transmitted can be verified by the client. It is not possible for the agent to accept the bribe, promising to lie and report the truth instead (that the project is bad), telling to the client afterwards that the manager has found out the project's real nature and has cancelled it. All the investment decisions are quite public in nature and cannot be concealed.

When the top manager has formal authority, if she finds a bad, she can stop its implementation, which is a typical case of the exercise of veto power.

\footnotetext{
${ }^{14}$ Since type 2 projects yield an expected negative payoff and the bribe is paid before the agent reports his information, the latter could cheat, take the bribe and then report the project as type 2, therefore stopping its implementation. However, this would jeopardize any future attempt to ask for a bribe, "spoiling " the agent's reputation.

${ }^{15}$ This case corresponds to the first best.
} 
Moreover, on the basis of that information, the manager can take the agent to court. The bribe is confiscated (a share $s \leq 1$ given to the top manager as a reward for cooperation) and the agent is fired..$^{16}$. If the agent is corrupt, the probability of being caught depends on the central office's effort and is equal to $e_{M}$.

When the agents have formal authority a bad project cannot be stopped (by the same definition of formal authority). However, if the top manager has evidence that the project is of bad, an agent can be convicted and the bribe confiscated. The manager will get a share $s b$ as a compensation.

Each agent's $h$ of being corrupted is distributed uniformly over the interval $[0,1]$. The higher $h$ the less prone to corruption an agent is.

The agent's gain from being corrupt is

$$
\Pi_{A}=\left(1-e_{M}\right)\left(b-g_{2}\right)-e_{M} z g_{2}-h
$$

if the agent decides to accept a bad project and the top manager has not the knowledge to stop him, the agent suffers the expected loss $g_{2}$, but gains the bribe $b$. When the manager has information and the project suggested is bad, the bribe is confiscated but the loss $g_{2}$ is born as long as the principal has not formal authority to stop the project.

From (2) it can be seen that a corrupt agent, if caught by the top manager, prefers to be stopped, that is he prefers not to have formal authority. This happens because a bribe here is paid just to induce him to lie, reporting false information to the top manager. When the agent is caught and the project stopped, although the bribe is confiscated, at least he does not bear the loss $g_{2}$.

A corrupt agent (characterized by $h \leq \tilde{h}$ ) has the following payoff

$$
U_{A}^{C}=\frac{g_{1}}{2}+\frac{1}{2} \Pi_{A}
$$

which, due to Assumption 2 and given that $\Pi_{A}>0$ for a corrupt agent, is always positive.

The manager chooses her monitoring effort after having observed the agent's report, which can either be "yes, the project is good" or "no, it should be discarded". Given the structure of the model, when a project is rejected it is never a good, as even the most corrupt agent would never discard a good. The manager never checks a negative report and monitors only the projects accompanied by a "yes" report.

\footnotetext{
${ }^{16}$ It could also be thought that the agent is jailed upon discovery of corruption. In that case, however, his payoff should include an additional loss term. To avoid making notation heavier, given that this would not enrich the results, the possibility of the agent being jailed is not considered.
} 
If the loss $G_{2}$ from a bad project is small enough, when the manager is not able to find any information about the suggested project, she prefers to rubberstamp the agent's suggestion rather than implementing no project and this regardless to the fact that the principal's loss $G$ can be rather high.

This requires that the expected return from a project chosen by an agent (who could be either honest or corrupt) is always positive, as the following assumption states

\section{Assumption $1 \frac{1}{1+\tilde{h}} G_{1}-\frac{\tilde{h}}{1+\tilde{h}} G_{2}>0$.}

Assumption 1 represents the top manager's expected payoff from a project recommended by an agent of unknown honesty. The probability that the project is good conditional to having been recommended is $\frac{1}{1+\tilde{h}}$ and a good project yields $G_{1}$. The conditional probability that the project is bad and yield $G_{2}$ is $\frac{\tilde{h}}{1+\tilde{h}} .17$ When Assumption 1 is satisfied, if the manager has not information, even if she has formal authority, the agent will actually take the final decision (exert real authority).

Given the diffusion of corruption in the hierarchy, $\tilde{h}$, and that all the agents exert positive effort, the top manager's payoff, conditional on a good report, is

$$
U_{M}=N\left(\frac{1+\tilde{h}}{2}\right)\left[\frac{G_{1}}{1+\tilde{h}}+\frac{\tilde{h}}{1+\tilde{h}}\left(e_{M} s b-\left(1-e_{M}(1-z)\right) G_{2}\right)-\frac{e_{M}^{2}}{2}\right]
$$

where $N\left(\frac{1+\tilde{h}}{2}\right)=N^{R}$, the number of recommended projects. The first term between square parentheses in (4) represents the expected gain if the project is good (probability of a good project conditional on being suggested per gain from a good project). The second term is the expected payoff if the project is bad. If the central office has neither information nor formal authority, she cannot overrule the agent who proposes a bad project. Therefore, she bears the expected loss $G_{2}$. Conversely, if she has information, she bears the loss only to the extent the agent has formal authority and cannot be stopped

\footnotetext{
${ }^{17}$ The conditional probability that a project is good given that it is recommended is obtained by Bayes rule $P($ good $\mid$ recomm $)=\frac{P(\text { good } \cap \text { recomm })}{P(\text { recomm })}$. A project is recommended either if it is good or if the agent is corrupt. Then $P($ recomm $)=\frac{1+\tilde{h}}{2}$. Given that all good projects are recommended, the joint probability that a project is good and recommended is $\frac{1}{2}$. Then $P($ good $\mid$ recomm $)=\frac{1}{1+\tilde{h}}$. Similarly, the conditional probability $P($ bad $\mid$ recomm $)=\frac{P(\text { badnrecomm })}{P(\text { recomm })}=\frac{\tilde{h}}{1+\tilde{h}}$ since only corrupt agents recommend bad projects and the probability that a agent is corrupt is $\tilde{h}$.
} 
(the share of control powers in the agent's hands is $z$ ). In any case, the top manager is rewarded with a share $s b$ of the bribe each time she denounces the agent, which happens if she is informed. The third term is the cost of monitoring. ${ }^{18}$

\section{Can decentralisation decrease corruption?}

The principal pursues the objective of reducing corruption. Therefore it will set the values of the policy tools at its disposal (namely, the degree of decentralization $z$ and the share $s$ to be transferred to the manager upon denunciation) to minimize equilibrium corruption ${ }^{19} \tilde{h}^{*}(z, s)$. After $z$ and $s$ have been chosen, production occurs: the agent reports his information and the manager verifies it. Solving this game backwards, the interaction between the manager and the agents is considered first.

As long as $\Pi_{A}>0$ in (2) the agent accepts the bribe. The value of $h$ that makes an agent just indifferent between being corrupt and not being corrupt, given $b$ and $e_{M}$ is found by solving $\Pi_{A}=0$ and is

$$
\tilde{h}=\left(b-g_{2}\right)-e_{M}\left(b-(1-z) g_{2}\right)
$$

The value $\tilde{h}$ represents a threshold such that all the agents with $h<\tilde{h}$ choose to be corrupt, whereas all those with $h>\tilde{h}$ prefer to remain honest. If $F(\tilde{h})=\operatorname{Pr}(h<\tilde{h})$ (in this case just equal to $\tilde{h}$ due to the uniform distribution over the interval $[0,1]), F(\tilde{h})$ represents also the share of corrupt agents, hence the diffusion of corruption.

\footnotetext{
${ }^{18}$ It is assumed here that the top bureaucrat obtains a reward if she reports a corrupt act to the police. This certainly enhances her incentives to work hard. However, this is not a crucial assumption, as it can be shown that the results obtained here would still hold if this assumption were removed and the bureaucrat did not obtain any compensation for reporting corrupt acts. In fact, checking expression (7), it can be seen that $e=\frac{\tilde{h}(1-z) G_{2}}{2 N}$, which is lower than the effort exerted when the reward is given, but qualitatively shows the same behaviour (increasing in $\tilde{h}$, decreasing in $z$ ). It has the (somehow undesirable) property of yielding an effort $e=0$ when $z=1$, as the manager has no incentives to monitor if she cannot intervene. To avoid this some kind of monetary incentives has to be introduced and bribe sharing is a possibility.

${ }^{19}$ The hypothesis that the principal minimizes corruption instead of maximizing a more general function measuring the welfare for the whole organization can be regarded as too simplistic. This hypothesis can be easily defended. First of all, minimization of corruption could be just one goal among many different ones. Moreover, as pointed out above, corruption entails a loss for the entire organization, therefore it is plausible to think that a function representing the organization's welfare should be decreasing in the diffusion of corruption. Finally, it can be shown that including a welfare function for the entire organization does not change the results dramatically.
} 
From (5), in order for $\tilde{h}$ to be positive, it has to be that

$$
e<\tilde{e}_{B}=\frac{b-g_{2}}{b-g_{2}(1-z)}<1
$$

Notice that $0<\tilde{e}_{B}<1$, if $b>g_{2}$ and given that $z<1$. Then, if the loss to the agent is larger than the bribe, collusion is not possible. Conversely, if $b$ is large, a relevant value $\tilde{e}_{B}$ always exists. We therefore introduce the following assumption.

Assumption $2 b>g_{2}$.

Direct observation of (5) reveals two important properties of this model.

Lemma 1. Given $e_{M}, \tilde{h}$ is decreasing in $z$.

Proof. From differentiation of expression (5), taking $e_{M}$ as given.

Conversely, it can be said that a decrease in the agent's formal authority (a decrease in $z$ ) increases corruption.

Lemma 2. As $e_{M}$ increases, $\tilde{h}$ decreases, reducing corruption.

Proof. By Assumption $2 b-g_{2}>0$, then $b-(1-z) g_{2}>0$, as $1-z<1$. Then an increase in $e_{M}$ always reduces $\tilde{h}$. Since the corrupt agents are those with $h<\tilde{h}$, this induces less agents to be corrupt. Moreover, if $H(\tilde{h})$ is the $\operatorname{Pr}(h<\tilde{h})$ (in this case just equal to $\tilde{h}$ due to the uniform distribution over the interval $[0,1])$ and the share of corrupt agent in the hierarchy, a decrease in the agent's real authority decreases corruption.

Given $z$ and $s$, from (4), the first order condition is ${ }^{20}$

$$
e_{M}=\frac{\tilde{h}}{1+\tilde{h}}\left[s b+(1-z) G_{2}\right]
$$

that reveals that $e_{M}$ is increasing in $\tilde{h}$, the diffusion of corruption in the society. Moreover, $e_{M}$ is increasing in the share of recommended projects $\frac{1+\tilde{h}}{2}$. This represents an overload effect. As the number of supervised projects increases (thus increasing the manager's span of control) the costs of monitoring them becomes higher and the manager's effort diminishes.

\footnotetext{
${ }^{20}$ It can be checked directly from (4) that the second order condition for a maximum is satisfied.
} 
Lemma 3. (i) The manager's reaction function $e(\tilde{h})$ is overall increasing and strictly concave. (ii) corruption as a function of $e, \tilde{h}(e)$, is discontinuous at $e=\tilde{e}_{B}$. It decreases linearly in $e_{M}$ for $e_{M} \in\left[0, \tilde{e}_{M}\right)$ and then reaches 0 for $e_{M} \in\left(\tilde{e}_{M}, 1\right]$.

Proof. Part (i) proved by differentiation of (7). Part (ii) follows from (5). When $e_{M}<\tilde{e}_{M}$, then $\frac{\partial \tilde{h}}{\partial e_{M}}<0$ by Lemma 2 and $\frac{\partial^{2} \tilde{h}}{\partial e_{M}^{2}}=2(1-z) v>0$. At $e_{M}=\tilde{e}_{M}, \tilde{h}\left(\tilde{e}_{M}\right)=0$ and when $e_{M}>\tilde{e}_{M}$ then $\tilde{h}<0$, so that, by definition of $\tilde{h}, \tilde{h}=0$.

The schedules $e_{M}(\tilde{h})$ and $\tilde{h}\left(e_{M}\right)$ are represented in Figure 2 below, where $\tilde{h}$ is measured on the vertical axis and $\phi\left(e_{M}\right)=e_{M}^{-1}(\tilde{h})$. The existence of a value $\tilde{e}_{M}$ for $e_{M}$ such that for $e_{M}>\tilde{e}_{M}$ there is no corruption means that there exist levels of monitoring effort in the admissible range such that corruption is inhibited. However, as it is also possible to infer from Figure 2, such levels of monitoring never occur in equilibrium. The following proposition formalises this result.

Proposition 1. If $b-g_{2}>0$, there exists a unique equilibrium $\left(e_{M}^{*}, \tilde{h}^{*}\right)$, with $e_{M}^{*} \in\left[0, \tilde{e}_{M}\right)$ and $\tilde{h}^{*} \in\left[0, \min \left\{b-g_{2}, 1\right\}\right]$.

Proof. Given that $b>g_{2}$ by Assumption 2, if $e_{M}=0, \tilde{h}(0)=\min \left\{b-g_{2}, 1\right\}$. By Lemma $3, \tilde{h}\left(e_{M}\right)$ is decreasing and convex for $e_{M} \in\left[0, \tilde{e}_{M}\right)$, at $\tilde{e}_{M}$ the schedule $\tilde{h}\left(e_{M}\right)$ reaches 0 . Conversely, the inverse of the manager's reaction function, $\tilde{h}_{M}^{-1}\left(e_{M}\right)$ is always increasing and convex in $e_{M}$. Therefore, the two schedules will cross at a point $\left(e_{M}^{*}, \tilde{h}_{M}^{*}\right)$, with $e_{M}^{*} \in\left[0, \tilde{e}_{M}\right)$ and $\tilde{h}^{*} \in$ $\left[0, \min \left\{b-g_{2}, 1\right\}\right]$.

Proposition 1 implies that if the bribe is high enough (or, conversely, if the loss due to a bad project is not too large), at the equilibrium detection is not enough to deter collusion, which then is always positive. Given the size of the losses $g_{2}$ and $G_{2}$, the bribe $b$ and the degree of decentralization $z$, this equilibrium is unique.

\section{Policies to reduce corruption}

At the stage before, the principal has to choose $z$ and $s, 0 \leq s, z \leq 1$ to minimize equilibrium corruption $\tilde{h}^{*}(z, s)$.

The following result is immediate to check.

Lemma 4. In equilibrium, for any $z, \tilde{h}^{*}(z, s)$ is always decreasing in $s$.

Proof. See Appendix. 
From the mere point of view of reducing corruption then, setting $s^{*}=1$ is optimal, even if, in a more general setting, the principal could prefer to reinvest a share of the proceeds from fighting corruption in, say, improving police equipment or hiring external auditors and detectives. ${ }^{21}$

Proposition 2. (i) Decentralisation always decreases the manager's effort in equilibrium. (ii) If $\frac{d \tilde{h}^{*}}{d z}<0$ then $\frac{\partial \tilde{h}^{*}}{\partial z}>\frac{\partial \tilde{h}^{*}}{\partial e_{M}} \frac{\partial e_{M}^{*}}{\partial z}$.

Proof. See Appendix.

According to Proposition 2, decentralisation always decreases the manager's effort in equilibrium. If this reduction in monitoring (hence in the probability of punishment) is large enough, the agent's accountability is lower and a more decentralized structure is also more corrupt. However, Lemma 2 shows that the direct impact of a reduction in $z$ on $\tilde{h}$ goes in the direction of reducing corruption. This is because the opportunity cost of recommending a bad project increases with monopoly power. If this second effect offsets the lower accountability, the power to decide discourages corruption.

In this section, subordinates are provided incentives by means of delegation of decisional power. It could also happen that the principal imposes a fine to the parties when they are caught being corrupt. ${ }^{22}$ Monetary incentives and penalties will be discussed more thoroughly in the next section.

Finally, work overload (namely, the increase in $N$, the number of agents monitored by the central office), reducing $e_{M}$, favors the diffusion of corruption in equilibrium. Increasing the central office's work overload always worsens the problem of corruption.

\section{Corruption and monetary incentives}

In this section, it will be shown that the model presented above is robust to the introduction of monetary incentive schemes and, specifically, that decentralization and monetary penalties have the same impact on corruption, being substitute policies.

\footnotetext{
${ }^{21}$ It can be shown that, from a quantitative point of view, a more general specification would entail a different value $0<\psi^{*}<1$ and a different $z$. Qualitatively, the conclusions presented in this section would be confirmed.

${ }^{22}$ The penalty is imposed only to the extent that a party has formal authority or, in the case of the central office, that she failed in collecting the evidence about the suggested project.
} 
The principal, when an agent is found out being corrupt and denounced by the central office, imposes a penalty on him. For a penalty equal to $\Pi$, the agent's expected payoff from corruption is

$$
M_{A}(P)=\left(1-e_{M}\right)\left(b-g_{2}\right)-e_{M}\left(z g_{2}+P\right)-h
$$

In order to discourage corruption, the principal should impose a penalty $P \geq P^{0}$ such that $M_{A}(P) \leq 0$. Given that the government does not observe $h$, which is private information for the agent, $P^{0}$ solves

$$
\left(1-e_{M}\right)\left(b-g_{2}\right)-e_{M}\left(z g_{2}+P^{0}\right)=0
$$

It can be verified from (9) that the minimum penalty to discourage corruption, $P^{0}$ can be decreased when the degree of decentralization, $z$ is higher. This implies that decentralization and monetary penalties ${ }^{23}$ are substitutes in fighting corruption: $z$ and $P$ are working in the same direction.

The existence of constitutional rules or laws may impose an upper bound to the maximum penalty applicable. For instance, a penalty may not be allowed to be higher than $\bar{P}$, where $\bar{P}<P^{0}$. In that case, punishment alone cannot minimize corruption and setting an appropriate organizational structure may help.

Another way of designing a penalty scheme could be that of considering $g_{2}$ and $G_{2}$ as fines imposed by the principal on the manager and on the agent when a bad project is implemented, fines that are proportional to the amount of formal authority held by each of them. Hence $g_{2}=g_{2}(z)$ and $G_{2}=G_{2}(1-z)$ and $g^{\prime}(z), G^{\prime}(1-z)>0 .^{24}$

In such case, the agent's benefit from corruption would be given again by (8), with the difference that, now, the loss $g_{2}$ depends on $z$.

Again, as in equilibrium there is no corruption, $e_{M}=\underline{e}_{M}$ and penalties and decentralization are substitutes. Decentralization helps in overcoming incentive problems due to the presence of limited liability constraints. ${ }^{25}$

\footnotetext{
${ }^{23}$ It has been chosen here to interpret $P$ as a monetary fine imposed on the agent. However, $P$ may represent any form of punishment that causes a loss for the agent, like being sent to jail.

${ }^{24} \mathrm{~A}$ simple functional form that embodies this idea could be linear, where $g_{2}(z)=z x L$ and $G_{2}(1-z)=(1-z) x L$, where $L$ is the loss the entire organization suffers as a consequence of a bad project and $x$ is the share of such loss that the principal demands the responsible to refund.

${ }^{25}$ A similar result can be found in Felli (1996), who, however, gives to the term "delegation" a very specific meaning. According to Felli, delegation is the transfer of (part) of the rights to be residual claimant from the principal to a subordinate layer. Felli then shows that, in a three-level hierarchy, (partial) delegation to the intermediate layer eases the incentive problems of both the subordinate layers, given that the intermediate layer is risk neutral.
} 
The results in this section can be reinterpreted in the light of the economic theory of the optimal enforcement of law (recently surveyed by Polinsky and Shavell, 1998). One of the main results of that theory is contained in Polinsky and Shavell (1979). They show that, when potential offenders are risk averse and the costs of catching them are high, there is a trade off between the probability of being caught (detection) and the magnitude of the punishment imposed. A very high punishment when detection is imperfect may have the perverse effect of increasing moral hazard in offenders. In our model, Polinsky and Shavell's trade off can be reinterpreted as follows. The amount of the penalty is directly proportional to the loss suffered when a bad project is chosen, whereas detection decreases with decentralization. Therefore, increasing decentralization increases the expected penalty (loss), at the same time lowering detection.

\section{A corrupt manager}

If the manager finds out that a project is bad and that the agent who recommended it is corrupt, she demands a side payment in order not to denounce him and, when she has formal authority, not to stop the project. If the payment is made, the project is completed and the agent retains his job. If the manager holds all the bargaining power, she will ask for the entire bribe paid to the agent, $b .{ }^{26}$ The agent prefers if the manager is corruptible. His bribe is confiscated and he always suffers the loss from a bad project but he does not lose his job. ${ }^{27}$

The timing of the game is modified as follows

1. the manager chooses $z$ and $s$

2. the agent is matched with a client

3. if bad, the client offers a bribe

4. the agent decides whether to accept and then reports to the manager the information he has, and, when he has formal authority, the decisions he has taken

5. the manager screens the information from all agents

\footnotetext{
${ }^{26}$ It can be checked that different distributions of the bargaining power would not change the results.

${ }^{27}$ In the incorruptible manager case, the agent bears $g_{2}$ only when he has formal authority, because in centralised decisional settings the manager would stop a bad project.
} 
6. if corruption is detected, the manager is paid the entire agent's bribe $b$, which, if accepted, guarantees project implementation ${ }^{28}$

7. selected projects are implemented.

Clearly, the manager is corrupt if the bribe is high enough to compensate her against all losses. Then the bribe $b$ must satisfy the following assump$\operatorname{tion}^{29}$

Assumption $1 \quad b>G_{2}$.

The manager's payoff is

$$
U_{M}^{C M}=N \frac{1+\tilde{h}}{2}\left[\frac{G_{1}}{1+\tilde{h}}+\frac{\tilde{h}}{1+\tilde{h}}\left(e_{M} b-G_{2}\right)-\frac{e_{M}^{2}}{2}\right]
$$

where the header $C M$ stands for "corrupt manager". The number of recommended projects ${ }^{30}$ is $N \frac{1+\tilde{h}}{2}$. Given that they are recommended, there is a conditional probability equal to $\frac{1}{1+\tilde{h}}$ that each of them is good and a conditional probability $\frac{\tilde{h}}{1+\tilde{h}}$ that each is bad. In this latter case, an informed manager gets the entire bribe $b$ (and not only a share $s$ of it), but also the loss $G_{2}$.

Differentiating (10), the first order condition for $e_{M}$ yields

$$
e_{M}^{C M}=\frac{\tilde{h}}{1+\tilde{h}} b
$$

which is increasing in $\tilde{h}$ and $b$. More interestingly, (11) is invariant with respect to $z$, as, no matter who is in control, a bad project, when recommended, always finds its way towards implementation and the loss $G_{2}$ is always born.

\footnotetext{
${ }^{28}$ Notice that here we consider only ex-post corruption, namely corruption takes place only after the manager monitored, once the agent has been discovered. There is also the possibility of ex-ante corruption, that is corruption before monitoring takes place. However, since trying to corrupt the manager would reveal the project's nature, the results and the size of the transfer would remain the same.

${ }^{29}$ It is assumed here that the manager "has always her own price", in the sense that any moral restraints or prestige considerations can be put aside in front of a generous enough offer. In the model economy here represented, this is not a crucial assumption. This is equivalent to assuming that the manager's private cost of corruption is constant and normalised to zero.

${ }^{30} \mathrm{~A}$ share $\frac{1+\tilde{h}}{2}$ of the $N$ available projects is recommended. All good projects are chosen $\left(\frac{N}{2}\right)$, plus all the bad ones matched with a corrupt agent $\left(\frac{N \tilde{h}}{2}\right)$.
} 
The agent's gain from corruption is

$$
\Pi_{A}^{C M}=b\left(1-e_{M}^{C M}\right)-g_{2}-h
$$

so that the threshold level $\tilde{h}$ is

$$
\tilde{h}^{C M}=b\left(1-e_{M}^{C M}\right)-g_{2}
$$

The agent accepts (hence $\tilde{h}^{C M}>0$ ), iff

$$
b\left(1-e_{M}^{C M}\right)>g_{2}
$$

The agent's gain from corruption is decreasing in the manager's monitoring effort: the detection effect still holds. However, as equation (13) reveals, now decentralisation has no direct effect on $\tilde{h}$, since $\tilde{h}^{C M}$ does not depend on $z$. The loss from a bad project is born in any case, because now the corrupt superior does not try to stop it. However, the full payment now is $b+b_{M}$.

If the manager is corrupt, decentralization tends to increase corruption. However, the minimum payment clients have to make to be sure their project is chosen is now larger. Therefore, only clients with high willingness to pay will be able to pay ${ }^{31}$ With respect to a situation with honest manager, the extent of corruption can thus be lower if $b_{M}$ is very large.

\section{When decentralization increases the bribe}

In this section we generalize the model introduced above, assuming that a change in the allocation of power also changes the amount the client is ready to pay. Usually, the bribe a client is willing to make is larger the higher the discretion of the agent he faces, for greater is the likelihood that he obtains what he is paying for. It is still assumed that the manager cannot be corrupted, so that corruption is ruled out.

Again, the manager and the agents are risk neutral. The bottom layer of the hierarchy consists of $N \geq 2$ agents.

Agents receive a private benefit $g_{1}$ from a good project and a loss $g_{2}$ from a bad one.

Each agent has a private-information cost $h$ of being corrupted, cost distributed uniformly in the population of agents over the interval $[0,1]$.

We summarise the model briefly, to stress the differences introduced in this new setting.

\footnotetext{
${ }^{31}$ A similar result can be found also in Banerjee (1997) and Carrillo (1995). Corruption can be socially detrimental as it allocates resources to and promotes individuals with the highest ability to pay, who are not necessarily the ones who deserve it.
} 
No corruption (first best) If all agents are honest, only good projects are implemented. In this case the results in Section 2.5 still hold. Given that (1) is everywhere decreasing in effort, the manager exerts only minimum effort $\underline{e}_{M} \geq 0$.

Corruption When corruption is possible, both good and bad projects are suggested, bad ones provided that the clients who propose them offer the agents a bribe $b$ sufficiently high. The bribe is paid to the agent to report the wrong information. Corruption then means that a bad project is presented as a good one to the superior.

When the manager has formal authority, if she finds a bad project, she can stop its implementation, which is a typical case of the exercise of veto power. On the basis of the same information, the manager can take the agent to court. The bribe is confiscated (a share $s \leq 1$ given to the manager as a reward for cooperation) and the agent is fired. If the agent is corrupt, the probability of being caught depends on the central office's effort and is equal to $e_{M}$. If the manager is not informed and Assumption 1 is satisfied, she accepts the agent's recommedation. Below, only cases where Assumption 1 holds will be considered.

When the agents have formal authority a bad project cannot be stopped (by the same definition of formal authority). However, if the manager has evidence that the project is bad, an agent can be convicted and the bribe confiscated. The manager will get a share $s b$ as a compensation.

A client with a bad project attributes a value $v$ to its implementation. His willingness to pay for corrupt services is therefore strictly linked to the probability of having his project chosen. This probability is increasing in the degree of decentralization $z$ and decreasing in the manager's effort $e_{M}$. If the agent has formal authority (which happens with probability $z$ ), the client is sure to obtain implementation. If the manager has formal authority (case with probability $1-z$ ), the client has his project chosen only if the manager is not informed, which, given her effort $e_{M}$ happens with probability $1-e_{M}$. Therefore, the client's willingness to pay is

$$
b=\left[1-e_{M}(1-z)\right] v
$$

The value $v$ is common knowledge and agents have all the bargaining power and can behave as perfectly discriminating monopolists, seizing the entire client's willingness to pay (15).

The agent's gain from being corrupt is given again by (2)

$$
\Pi_{A}=\left(1-e_{M}\right)\left(b-g_{2}\right)-e_{M} z g_{2}-h
$$


Substituting $b(z)$ from (15) in the expression above and rearranging terms, the agent's gain from corruption becomes

$$
\Pi_{A}=\left[1-e_{M}(1-z)\right]\left[\left(1-e_{M}\right) v-g_{2}\right]-h
$$

As long as $\Pi_{A}>0$, the agent accepts the bribe. From (16), the value of $h$ that makes an agent just indifferent between being corrupt and not being corrupt, given $b$ and $e_{M}$ is

$$
\tilde{h}=\left[1-e_{M}(1-z)\right]\left[\left(1-e_{M}\right) v-g_{2}\right]
$$

All agents with $h<\tilde{h}$ choose to be corrupt, whereas all those with $h>\tilde{h}$ prefer to remain honest.

In order for $\tilde{h}$ to be positive in equilibrium, given that $1-e_{M}(1-z)>0$, the term $\left[\left(1-e_{M}\right) v-g_{2}\right]$ in (17) has to be positive too, i.e.

$$
e_{M}<\tilde{e}_{M}^{\prime}=\frac{v-g_{2}}{v}
$$

Notice that $0<\tilde{e}_{M}^{\prime}<1$ if $v>g_{2}$. Then, if the loss to the agent is larger than the client's value of the project, corruption is not possible. Conversely, if $v$ is large, a relevant value $\tilde{e}_{M}^{\prime}$ always exists.

Direct observation of (17) leads to the following result.

Lemma 5. Given $e_{M}, \tilde{h}$ (hence the diffusion of corruption) is increasing in $z$.

Proof. Straightforward, from differentiation of (5) with respect to $z$, given that the inequality in (18) holds.

The Lemma above reverts the result in Lemma 1. This means that when the bribe increases with decentralization more agent's formal authority increases corruption.

The manager chooses her monitoring effort $e_{M}$ after having observed the agent's report.

The manager's payoff, conditional on a good report is still given by expression (4)

$$
U_{B}=\frac{N}{2}(1+\tilde{h})\left\{\left(1-\frac{\tilde{h}}{2}\right) G_{1}+\frac{\tilde{h}}{2}\left[e_{M} s b-\left(1-e_{M}(1-z)\right) G_{2}\right]-\frac{e_{M}}{2}\right\}
$$

The manager is rewarded with a share $s b$ of the bribe if she denounces the agent. We still assume that the manager is incorruptible. Therefore, any time she is informed and the project is bad she reports that corruption occurred. 
Given $z$ and $s$, set by the principal in the first stage of the game, the manager's first order condition is

$$
\frac{\tilde{h}\left(s b+(1-z) G_{2}\right)}{2}-e_{M}=0
$$

Substituting the above expression for the expected bribe $b$ from (15) and solving for $e_{M}$, we find the manager's reaction function to $\tilde{h}$ that takes into account the changes in the expected bribe due to changes in monitoring effort

$$
e_{M}(\tilde{h})=\frac{\tilde{h}\left(s v+(1-z) G_{2}\right)}{2+s v \tilde{h}(1-z)}
$$

It is possible to show that the results in Lemma 3 and Proposition 1 still hold.

Lemma 6. (i) The manager's reaction function $e_{M}(\tilde{h})$ is overall increasing and strictly concave. (ii) corruption as a function of $e_{M}, \tilde{h}\left(e_{M}\right)$, is discontinuous at $e_{M}=\tilde{e}_{B}$. It is decreasing and convex for $e_{M} \in\left[0, \tilde{e}_{B}\right)$ and then jumps to 0 for $e_{M} \in\left(\tilde{e}_{B}, 1\right]$.

Proof. See Appendix.

Proposition 3. If $v-g_{2}>0$, there exists a unique equilibrium $\left(e_{M}, \tilde{h}^{*}\right)$, with $e_{M} \in\left[0, \tilde{e}_{B}\right)$ and $\tilde{h}^{*} \in\left[0, \min \left\{v-g_{2}, 1\right\}\right]$.

Proof. See Appendix.

The main issue we want to investigate in this section is how equilibrium corruption changes with decentralisation now that the bribe increases with $z$.

Lemma 5 has shown that, for given $e_{M}$, an increase in $z$ increases corruption. We now check whether this result still holds in equilibrium, when $e_{M}$ too changes.

Differentiating the manager's reaction function given by (19) with respect to $z$ the following can be proved.

Lemma 7. Given $\tilde{h}$, the manager decreases her effort $e_{M}$ when $z$ increases if and only if $\tilde{h}<\tilde{h}_{G}=\frac{2 G_{2}}{s^{2} v^{2}}$.

Proof. Straightforward, by differentiation of (19) with respect to $z$. 
Lemma 7 states that the manager exerts less effort following decentralization if corruption is low. More decentralization causes a loss in control to the manager, who now is less able to interfere with the agent's decisions, hence reduces her incentive to exert effort and bear the cost of it. This is more likely to happen when $G_{2}$, the loss the manager suffers from a bad project, is high relative to the share of the bribe she receives if she discovers and reports corruption, $s v$. Therefore, when $G_{2}$ is high, the basic trade off dominates and decentralization decreases the manager's effort for any level of corruption, $\tilde{h}$.

Conversely, if $\tilde{h}>\tilde{h}_{G}=\frac{2 G_{2}}{s^{2} v^{2}}$, following decentralization, the manager's reaction function moves downward, implying a higher effort $e_{M}$ for any level of $\tilde{h}$. In this second case, what we could term bribe-confiscation effect dominates. This result is new and did not exist in the previous setting. It is due to the increase in the size of the bribe caused by decentralisation, that induce the manager to increase effort to receive a higher compensation. This result reverts Aghion and Tirole's (1997) basic trade off, according to which delegation discourages the superior's initiative, enhancing the subordinate's effort. If the bribe increases enough, the manager may put considerably more effort in monitoring, thus leading to a decrease in corruption following decentralisation . ${ }^{32}$

Proposition 4. (i) When $\frac{\partial e_{M}}{\partial z}<0$, in equilibrium decentralization always increases corruption, whereas $\frac{d e_{M}}{d z}$ is either positive (if the basic trade off dominates) or negative (if the bribe-confiscation effect is relatively stronger).

(ii) When $\frac{\partial e_{M}}{\partial z}>0$, in equilibrium the bribe-confiscation effect dominates and $\frac{d e_{M}}{d z}>0$. corruption can either increase or decrease with decentralization.

Proof. See Appendix.

If the bribe-confiscation effect is strong enough, the agent's expected decreases with decentralization and corruption is lower.

This result is rather counterintuitive, as it would be more straightforward to figure out a situation where more decisional power to the people in direct contact with bribers implies both a higher expected bribe and more propensity to corruption. The key role here is played by the manager's incentives

\footnotetext{
${ }^{32}$ To show that the sign of $\frac{\partial e_{B}}{\partial z}$ depends on the relative size of the basic trade off vs the bribe confiscation effect, totally differentiate expression (7), that gives $e_{B}$ for given $\tilde{h}$ and b. Obtain $\frac{d e_{B}}{d z}\left(2-\tilde{h} s \frac{\partial b}{\partial e_{B}}\right)=\tilde{h} s \frac{\partial b}{\partial z}-2 G_{2}$. The l.h.s. is positive, since $\frac{\partial b}{\partial e_{B}}<0$. The r.h.s. has an ambiguous sign. If the bribe confiscation effect $\tilde{h} s \frac{\partial b}{\partial z}>0$ (recall $\frac{\partial b}{\partial z}>0$ ) is larger than the basic trade off effect $G_{2}$, then $\frac{\partial e_{B}}{\partial z}>0$ and vice-versa.
} 
to monitor. If her reward increases with the number and amount of reported bribes, decentralization can prove an effective device to fight corruption.

Notice that this conclusion still holds if the assumption that the manager is incorruptible is released. In such case, the manager is still able to seize a share of the bribe paid to the agent, but the amount of this share depends on her bargaining power vis à vis the agent. In any case, for corruption to be possible it must be $r>s$, where $r$ is the share the manager obtains in case she colludes. ${ }^{33}$ Again decentralization, increasing the client's willigness to pay, triggers the bribe-confiscation effect and can curb corruption. This last effect is even stronger in the event of corruption (being $r>s$ ), however the desirability of decentralization can be lower from a social point of view than in the no corruption case. In fact, resources are shared among the client, the agent and the manager and there is no share $1-s$, however small, that is redistributed to society.

\section{Conclusions}

This paper has analyzed the effects of decentralization on the extent of corruption in a hierarchical organization, where decentralization here has been defined as the delegation of control power to lower layers in the hierarchy. The principal stipulates a grand-contract with all the subordinates in each layer deciding how much control power to delegate them (all individuals placed at the same level receive the same contract) and how to compensate them if they report acts of corruption committed by their direct subordinates.

When decentralization is high, the low levels of an organization have more discretion and are freer to supply illegal services, without having to worry too much about being overruled by superiors. However, this does not lead automatically to the conclusion that decentralization leads to more corruption. Decentralization causes a trade off between the superiors' incentives to monitor and the agent's willingness to bear the (increasing) costs from corruption. According to which aspect prevails in this trade off, more centralized and complex structures can be affected by corruption to a greater extent, independently of the old argument that more complex organizations suffer more from problems of corruption because of conditions of work overload. Superiors have to supervise a large number of agents, which certainly reduces the amount of time and resources that can be spent on monitoring each single agent.

\footnotetext{
${ }^{33}$ The share $r$ must be strictly larger than $s$ because it is plausible that, when the top bureaucrat is indifferent between colluding and being honest, she would rather be honest.
} 
If the superior layers in charge of detecting and punishing corruption are corrupt themselves, decentralisation actually increases corruption. However, it also make it more expensive for corruptors to obtain what they want, thus narrowing the set of people who can afford it.

If the bribe increases with decentralization, the larger expected loss to an agent due to a higher probability that his choices go through is compensated by the higher bribe. However, it is still the case that decentralisation reduces the extent of corruption at the condition that the manager's payoff is directly proportional to the amount of bribes confiscated to agents. This assumption is quite plausible even without assuming that a certain share of these bribes is actually paid to her. It is easy to imagine that her reputation improves substantially if she discovers serious cases of corruption, so that she derives a higher private benefit. ${ }^{34}$

If the manager benefits more from uncovering more bribes, decentralization can improve her incentives to monitor corrupt agents, thus increasing the probability that they are caught and the expected costs from corruption and it is again possible that decentralisation helps in controlling corruption.

\section{A Appendix A: Proofs}

\section{A.0.1 Proof of Lemma 4}

By the implicit function theorem, the impact of a change in $s$ on equilibrium $e_{M}^{*}$ is

$$
\frac{d e_{M}^{*}}{d s}=\frac{\partial e_{M}^{*}}{\partial s}+\frac{\partial e_{M}^{*}}{\partial \tilde{h}} \frac{d \tilde{h}^{*}}{d s}
$$

whereas the impact on equilibrium corruption is

$$
\frac{d \tilde{h}^{*}}{d s}=\frac{\partial \tilde{h}^{*}}{\partial e_{M}} \frac{d e_{M}^{*}}{d s}
$$

as the direct effect $\frac{\partial \tilde{h}^{*}}{\partial s}=0$. If there is corruption in the system $\left(\right.$ i.e. $\left.e_{M}^{*}<\tilde{e}_{M}\right)$ then, by Lemma $2, \frac{\partial \tilde{h}^{*}}{\partial e_{M}}<0$. Therefore (21) reveals that $\frac{d \tilde{h}^{*}}{d s} \gtrless 0$ iff $\frac{d e_{M}^{*}}{d s} \lessgtr 0$.

\footnotetext{
${ }^{34}$ In Italy, during the "clean hands" phase, many magistrates were involved in the investigation and prosecution of corrupt acts. However, it was those who handled the most serious cases, where huge bribes had been exchanged, who got the highest benefits, in terms of popularity, promotions and successful entry into the political stage.
} 
The sign of $\frac{d e_{M}^{*}}{d s}$ depends entirely on the sign of $\frac{\partial e_{M}^{*}}{\partial s}$. To show this, substitute (21) into (20) and solve out for $\frac{d e_{M}^{*}}{d s}$

$$
\frac{d e_{M}^{*}}{d s}=\frac{\frac{\partial e_{M}^{*}}{\partial s}}{1-\frac{\partial e_{M}^{*}}{\partial \tilde{h}} \frac{\partial \tilde{h}^{*}}{\partial e_{M}}}
$$

Notice that $1-\frac{\partial e_{M}^{*}}{\partial \tilde{h}} \frac{\partial \tilde{h}^{*}}{\partial e_{M}}>0$, as $\frac{\partial e_{M}^{*}}{\partial \tilde{h}}>0$ by Lemma 3 and $\frac{\partial \tilde{h}^{*}}{\partial e_{M}}<0$. Therefore $\operatorname{sign}\left[\frac{d e_{M}^{*}}{d s}\right]=\operatorname{sign}\left[\frac{\partial e_{M}^{*}}{\partial s}\right]$. Then we have proved that $\frac{d \tilde{h}^{*}}{d s}<0$, given that $\frac{\partial e_{M}^{*}}{\partial s}>0$.

\section{A.0.2 Proof of Proposition 2}

By the implicit function theorem, the effect of a change in $z$ on equilibrium effort can be written

$$
\frac{d e_{M}^{*}}{d z}=\frac{\partial e_{M}^{*}}{\partial z}+\frac{\partial e_{M}^{*}}{\partial \tilde{h}} \frac{d \tilde{h}^{*}}{d z}
$$

where $\frac{\partial e_{M}^{*}}{\partial z}$ is the direct effect and is negative whereas $\frac{\partial e_{M}^{*}}{\partial \tilde{h}}$ is the manager's reaction to an increase in the expected level of corruption and is positive by Lemma 3.

The effect of a change in $z$ on equilibrium corruption, always by the implicit function theorem, is

$$
\frac{d \tilde{h}^{*}}{d z}=\frac{\partial \tilde{h}^{*}}{\partial z}+\frac{\partial \tilde{h}^{*}}{\partial e_{M}} \frac{d e_{M}^{*}}{d z}
$$

where $\frac{\partial \tilde{h}^{*}}{\partial z}<0$ by Lemma 1 and $\frac{\partial \tilde{h}^{*}}{\partial e_{M}}<0$ by Lemma 2 .

Solving (23) and (24) $\frac{d e_{M}^{*}}{d z}$ is

$$
\frac{d e_{M}^{*}}{d z}=\frac{\frac{\partial e_{M}^{*}}{\partial z}+\frac{\partial e_{M}^{*}}{\partial \tilde{h}} \frac{\partial \tilde{h}^{*}}{\partial z}}{1-\frac{\partial e_{M}^{*}}{\partial \tilde{h}} \frac{\partial \tilde{h}^{*}}{\partial e_{M}}}
$$

and

$$
\frac{d \tilde{h}^{*}}{d z}=\frac{\frac{\partial \tilde{h}^{*}}{\partial z}+\frac{\partial \tilde{h}^{*}}{\partial e_{M}} \frac{\partial e_{M}^{*}}{\partial z}}{1-\frac{\partial e_{M}^{*}}{\partial \tilde{h}} \frac{\partial \tilde{h}^{*}}{\partial e_{M}}}
$$

Notice that $1-\frac{\partial e_{M}^{*}}{\partial \tilde{h}} \frac{\partial \tilde{h}^{*}}{\partial e_{M}}>0$ always. In (25), $\frac{\partial e_{M}^{*}}{\partial \tilde{h}} \frac{\partial \tilde{h}^{*}}{\partial z}<0$. Therefore, given that $\frac{\partial e_{M}^{*}}{\partial z}<0$ (basic trade off), $\frac{d e_{M}^{*}}{d z}<0$ always. This proves part (i) of Proposition 2. 
In (26), $\frac{\partial e_{M}^{*}}{\partial z}<0$, then $\frac{\partial \tilde{h}^{*}}{\partial e_{M}} \frac{\partial e_{M}^{*}}{\partial z}>0$. Thus, if $\frac{\partial \tilde{h}^{*}}{\partial z}<\frac{\partial \tilde{h}^{*}}{\partial e_{M}} \frac{\partial e_{M}^{*}}{\partial z}$ (basic trade off dominates) then $\frac{d \tilde{h}^{*}}{d z}>0$ and equilibrium corruption increases with decentralization. If $\frac{\partial \tilde{h}^{*}}{\partial z}>\frac{\partial \tilde{h}^{*}}{\partial e_{M}} \frac{\partial e_{M}^{*}}{\partial z}$ then $\frac{d \tilde{h}^{*}}{d z}<0$ and corruption decreases. This proves part (ii).

\section{A.0.3 Proof of Lemma 6}

The first part of Lemma 5 is proved by differentiation of (19). The second part follows from (17). When $e_{M}<\tilde{e}_{M}$, then $\frac{\partial \tilde{h}}{\partial e_{M}}<0$ from (17) and $\frac{\partial^{2} \tilde{h}}{\partial e_{M}^{2}}=$ $2(1-z) v>0$. At $e_{M}=\tilde{e}_{M}, \tilde{h}\left(\tilde{e}_{M}\right)=0$ and when $e_{M}>\tilde{e}_{M}$ then $\tilde{h}<0$, so that, by definition of $\tilde{h}, \tilde{h}=0$.

\section{A.0.4 Proof of Proposition 3}

If $v-g_{2}<0$ then $\tilde{h}<0$, then, by definition of $\tilde{h}, \tilde{h}=0$ and corruption is not possible. Assume $v-g_{2}>0$. Then, if $e_{M}=0, \tilde{h}(0)=\min \left\{v-g_{2}, 1\right\}$. From (17) $\tilde{h}\left(e_{M}\right)$ is decreasing and convex for $e_{M} \in\left[0, \tilde{e}_{M}\right)$, at $\tilde{e}_{M}$ there is a discontinuity, so that $\tilde{h}\left(e_{M}\right)$ jumps to 0 . Conversely, the inverse of the manager's reaction function, $\tilde{h}_{M}^{-1}\left(e_{M}\right)$ is always increasing and convex in $e_{M}$. Therefore, the two schedules will cross at a point $\left(e_{M}^{*}, \tilde{h}^{*}\right)$, with $e_{M}^{*} \in\left[0, \tilde{e}_{M}\right)$ and $\tilde{h}^{*} \in\left[0, \min \left\{v-g_{2}, 1\right\}\right]$.

\section{A.0.5 Proof of Proposition 4}

By the implicit function theorem, the effect of a change in $z$ on equilibrium effort can be written

$$
\frac{d e_{M}^{*}}{d z}=\frac{\partial e_{M}^{*}}{\partial z}+\frac{\partial e_{M}^{*}}{\partial \tilde{h}} \frac{d \tilde{h}^{*}}{d z}
$$

where $\frac{\partial e_{M}^{*}}{\partial z}$ is the direct effect and is negative when $\tilde{h}<\tilde{h}_{G}$, positive otherwise, by Lemma 8 and $\frac{\partial e_{M}^{*}}{\partial \tilde{h}}$ is the reaction of the manager to an increase in the expected level of corruption in the organization and is positive by Lemma 6 .

The effect of a change in $z$ on equilibrium corruption, always by the implicit function theorem, is

$$
\frac{d \tilde{h}^{*}}{d z}=\frac{\partial \tilde{h}^{*}}{\partial z}+\frac{\partial \tilde{h}^{*}}{\partial e_{M}} \frac{d e_{M}^{*}}{d z}
$$

where $\frac{\partial \tilde{h}^{*}}{\partial z}>0$ by Lemma 5 and $\frac{\partial \tilde{h}^{*}}{\partial e_{M}}<0$ if $e_{M}<\tilde{e}_{M}$. 
Solving (27) and (28) $\frac{d e_{M}^{*}}{d z}$ is

$$
\frac{d e_{M}^{*}}{d z}=\frac{\frac{\partial e_{M}^{*}}{\partial z}+\frac{\partial e_{M}^{*}}{\partial \tilde{h}} \frac{\partial \tilde{h}^{*}}{\partial z}}{1-\frac{\partial e_{M}^{*}}{\partial \tilde{h}} \frac{\partial \tilde{h}^{*}}{\partial e_{M}}}
$$

and

$$
\frac{d \tilde{h}^{*}}{d z}=\frac{\frac{\partial \tilde{h}^{*}}{\partial z}+\frac{\partial \tilde{h}^{*}}{\partial e_{M}} \frac{\partial e_{M}^{*}}{\partial z}}{1-\frac{\partial e_{M}^{*}}{\partial \tilde{h}} \frac{\partial \tilde{h}^{*}}{\partial e_{M}}}
$$

Notice that $1-\frac{\partial e_{M}^{*}}{\partial \tilde{h}} \frac{\partial \tilde{h}^{*}}{\partial e_{M}}>0$ always. From (30), if $\frac{\partial e_{M}^{*}}{\partial z}<0$, then $\frac{\partial \tilde{h}^{*}}{\partial e_{M}} \frac{\partial e_{M}^{*}}{\partial z}>$ 0 . Thus, if $\frac{\partial e_{M}^{*}}{\partial z}<0, \frac{d \tilde{h}^{*}}{d z}>0$ always. Equilibrium corruption increases with decentralization.

In (29), $\frac{\partial e_{M}^{*}}{\partial \tilde{h}} \frac{\partial \tilde{h}^{*}}{\partial z}>0$. Therefore, if $\tilde{h}<\tilde{h}_{G}$, so that $\frac{\partial e_{M}^{*}}{\partial z}<0$ (the basic trade off dominates), sign $\left[\frac{d e_{M}^{*}}{d z}\right]$ is ambiguous, positive if $\left|\frac{\partial e_{M}^{*}}{\partial z}\right|$ is small, negative if $\left|\frac{\partial e_{M}^{*}}{\partial z}\right|$ is large enough. This proves part $(i)$ of Proposition 4.

Conversely, if $\tilde{h}>\tilde{h}_{G}$, then $\frac{\partial e_{M}^{*}}{\partial z}>0$ (the bribe-confiscation effect dominates). In that case, sign $\left[\frac{d e_{M}^{*}}{d z}\right]>0$ always. According to (30), this leads to a decrease in equilibrium corruption iff $\left|\frac{\partial \tilde{h}^{*}}{\partial e_{M}} \frac{d e_{M}^{*}}{d z}\right|>\left|\frac{\partial \tilde{h}^{*}}{\partial z}\right|$. This proves part (ii).

\section{References}

[1] Acemoglu, D. and T. Verdier (1997), The Choice between Market Failures and Corruption, Document de travail $n^{\circ}$ 97-06, Delta, Paris.

[2] Aghion, P. and P. Bolton (1992), An Incomplete Contract Approach to Financial Contracting, RES, 59(3), 473-494.

[3] Aghion, P. and J.Tirole (1997), Formal and Real Authority in Organizations, JPE, 105(1), 1-29.

[4] Bac, M. (1996), Corruption, and the Structure of Hierarchies, J. of Law, Econ. and Org, 12(2), 277-98.

[5] Banerjee, A.V. (1997), A Theory of Misgovernance, QJE, 112, 12891332. 
[6] Bardhan, P. (1997), Corruption and Development:a Review of Issues, JEL, vol. $X X X V$ (September), 1320-1346.

[7] Baron, D.P. and D. Besanko (1984), "Regulation, Asymmetric Information and Auditing", Rand J. of Econ., 15(4): 447-470.

[8] Basu, K., S. Bhattacharya and A.Mishra (1992), Notes on Bribery and the Control of Corruption, J. Public Econ., 48, 349-359.

[9] Becker, G. S. and G.J. Stigler (1974), Law Enforcement, Malfeasance, and the Compensation of Enforcers, J. Legal Stud., vol. III, 1-19.

[10] Burkart, M., D.Gromb and F.Panunzi (1997), Large Shareholders, Monitoring and the Value of the Firm, QJE, 112, 693-728.

[11] Cadot, O. (1987), Corruption as a Gamble, J. Public Econ., 33(2), 223244.

[12] Carrillo, J.D. (1995), Grafts, Bribes and the Practice of Corruption, mimeo, GREMAQ, Toulouse.

[13] Carrillo, J.D. (1995), Corruption in Hierarchies, mimeo, GREMAQ, Toulouse.

[14] Felli, L. (1996), Preventing Collusion Through Discretion, London School of Economics, STICERD Discussion Paper Series TE/96/303, March.

[15] Klitgaard (1988), Controlling Corruption, Berkeley, University of California Press.

[16] Kofman, F. and J. Lawarrée (1993), Collusion in Hierarchical Agency, Econometrica, 61, 629-656.

[17] Laffont, J-J. and J. Tirole (1993), A Theory of Incentives in Procurement and Regulation, The MIT Press, Cambridge, Massachusets.

[18] Mauro, P. (1995), Corruption and Growth, QJE, 110(3), 681-712.

[19] Mookherjee, D. and I.P. Png (1995), Corruptible Law Enforcers: How Should They be Compensated, Econ. J., 105(428), 145-159.

[20] Polinsky, A.M. and S. Shavell (1979), The Optimal Tradeoff between the Probability and Magnitude, AER., 69(5): 880-891. 
[21] Polinsky, A.M. and S. Shavell (1998), The Economic Theory of Public Enforcement of Law

[22] Rose-Ackerman, S. (1978), Corruption: A Study in Political Economy, Academic Press, New York.

[23] Sah, R. and J. Stiglitz (1986), The Architecture of Economic Systems: Hierarchies and Polyarchies, AER, 76, 716-727.

[24] Shleifer, A. and R.W. Vishny (1993), Corruption, QJE, 108(3), 599-617.

[25] Tanzi, V. (1998), Corruption Around the World: Causes, Consequences, Scope, and Cures, IMF Working Paper, no. WP/98/63, Washington, May.

[26] Tanzi, V. and H.Davoodi (1997), Corruption, Public Investment, and Growth, IMF Working Paper, no. WP/97/139, Washington, October.

[27] Tirole, J. (1986), Hierarchies and Bureaucracies: on the Role of Collusion in Organizations, J. Law, Econ. Org., 2, 181-214.

[28] Tirole, J. (1996), A Theory of Collective Reputation (with applications to the persistence of corruption and to firm quality), RES, 63(1), 1-22. 


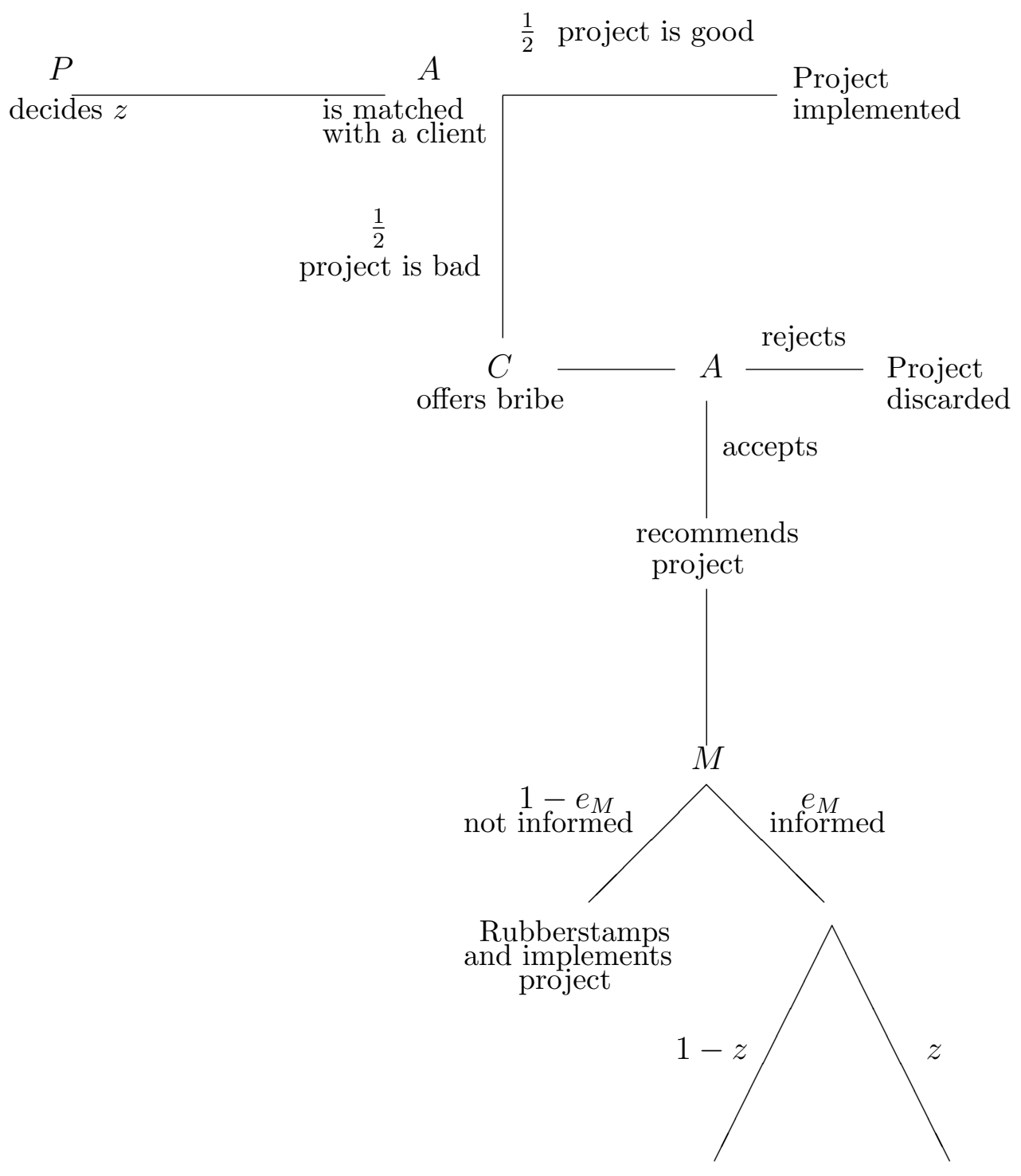

Project stopped Project goes ahead Agent punished Agent punished

Figure 1:Timing of the game 
Figure 2:Equilibrium Corruption

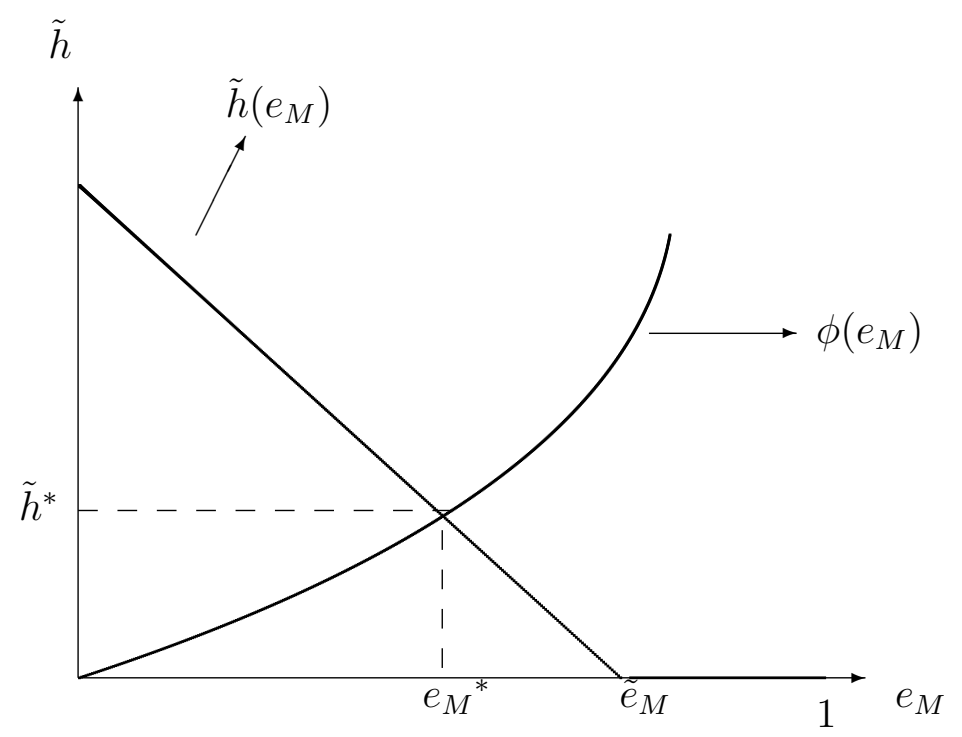

